\title{
Overview of transverse spin physics in the PHENIX experiment
}

\author{
Marie Boër*t \\ Los Alamos National Laboratory, \\ E-mail: mboerarcf.rhic.bnl.gov
}

\begin{abstract}
We present an overview of recent results from the PHENIX collaboration on spin physics using a transversely polarized proton beam. Several experiments have been conducted at RHIC in the past decade using such beam polarization in proton-proton collisions. Recently, polarized protonnucleus collisions have also been studied. Important recent transverse spin measurements in PHENIX include the transverse spin asymmetry in light meson production $\left(\pi^{0}, \eta\right)$, where a nonzero asymmetry can be interpreted as an initial state interaction (related to the Sivers effect) or a final state correlation (partially related to the Collins effect). Transverse spin asymmetries have

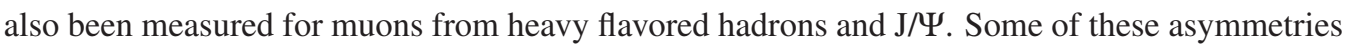
have been measured in both proton-proton and proton-ion collision allowing the study of nuclear dependence.
\end{abstract}

XXV International Workshop on Deep-Inelastic Scattering and Related Subjects

3-7 April 2017

University of Birmingham, $U K$

\footnotetext{
* Speaker.

${ }^{\dagger}$ for the PHENIX collaboration
} 


\section{Introduction: transverse spin physics in the PHENIX experiment}

\subsection{Physic interests}

One major interest of measurements using transversely polarized colliding beams is to probe the transverse structure of the nucleon (see for instance review from [1]). Indeed, several nonperturbative effects coming from quark and gluon dynamics inside the nucleon may induce nonzero spin asymmetries when using transversely polarized protons. Such asymmetries are defined as:

$$
A_{N}=\frac{\sigma^{\uparrow}-\sigma^{\downarrow}}{\sigma^{\uparrow}+\sigma^{\downarrow}}
$$

where $\sigma^{\uparrow, \downarrow}$ represent polarized cross sections with a proton spin pointing along up and down respectively of the $\mathrm{x}$-axis defined to be perpendicular to the beam direction.

Effects inducing non-zero spin asymmetries in $p+p$ collisions include the Sivers effect, the Collins effect, and other interaction effects involving gluon exchange at initial and/or final states (sub-leading twist effects) [2, 3, 4]. The well-known Sivers effect comes from the correlation between the proton transverse spin and the quark angular motion, and is interpreted as an initial effect in hadrons collisions. The Collins effect is interpreted as a final state effect where a non zero asymmetry is induced by the asymmetric fragmentation of polarized quarks, having a non zero transverse momentum $k_{T}$, into hadrons. The PHENIX measurements reported here observe only a single scale and need to be interpreted in the collinear factorization framework as initial/final state higher twist effects.

\subsection{Experimental conditions}

The PHENIX experiment benefits from the RHIC proton-proton and proton-ion beams. Since 2002, several runs have been taken using a transversely polarized proton beam, allowing for measurement of transverse spin asymmetries [5]. Over $90 \mathrm{pb}^{-1}$ of transversely polarized $\mathrm{p}+\mathrm{p}$ data have been accumulated with an average polarization ranging from $\sim 50 \%$ to $\sim 60 \%$. In 2015 , for the first time, $3.97 \mathrm{pb}^{-} 1$ and $1.27 \mathrm{pb}^{-} 1$ respectively of $\mathrm{p}+\mathrm{Al}$ and $\mathrm{p}+\mathrm{Au}$ data have been taken, where the proton was transversely polarized (with an average polarization of $\sim 54 \%$ and $\sim 60 \%$ in $\mathrm{p}+\mathrm{Al}$ and $\mathrm{p}+\mathrm{Au}$, respectively). This allows for the first time to study nuclear effects in transversely polarized spin asymmetries. All but the 2006 run with transverse proton polarization were at a beam center of mass energy of $\sqrt{s}=200 \mathrm{GeV}(\sqrt{s}=62.4 \mathrm{GeV}$ for the 2006 run $)$.

The PHENIX spectrometer [6] allows for measurements at both mid rapidity and forward rapidities. Charged and neutral hadrons, with hadron identification, are reconstructed in the central region of the PHENIX spectrometer in a rapidity region $|\eta|<0.35$. These measurements are used for neutral mesons $\left(\pi^{0}, \eta\right)$ analysis presented in this proceeding for the low rapidity region. At forward rapidity $(1.2<|\eta|<2.4)$ muons are reconstructed in two muon arms, working as two independent spectrometers in the backward and forward regions. The heavy quark and $J / \Psi$ analyses presented in this proceeding have been performed in this region of PHENIX spectrometer. At even larger rapidity $(|\eta|>3.1)$, neutral meson measurements $\left(\pi^{0}, \eta\right)$ were also performed. 


\section{Transverse spin asymmetries of light mesons: $\pi^{0}$ and $\eta$}
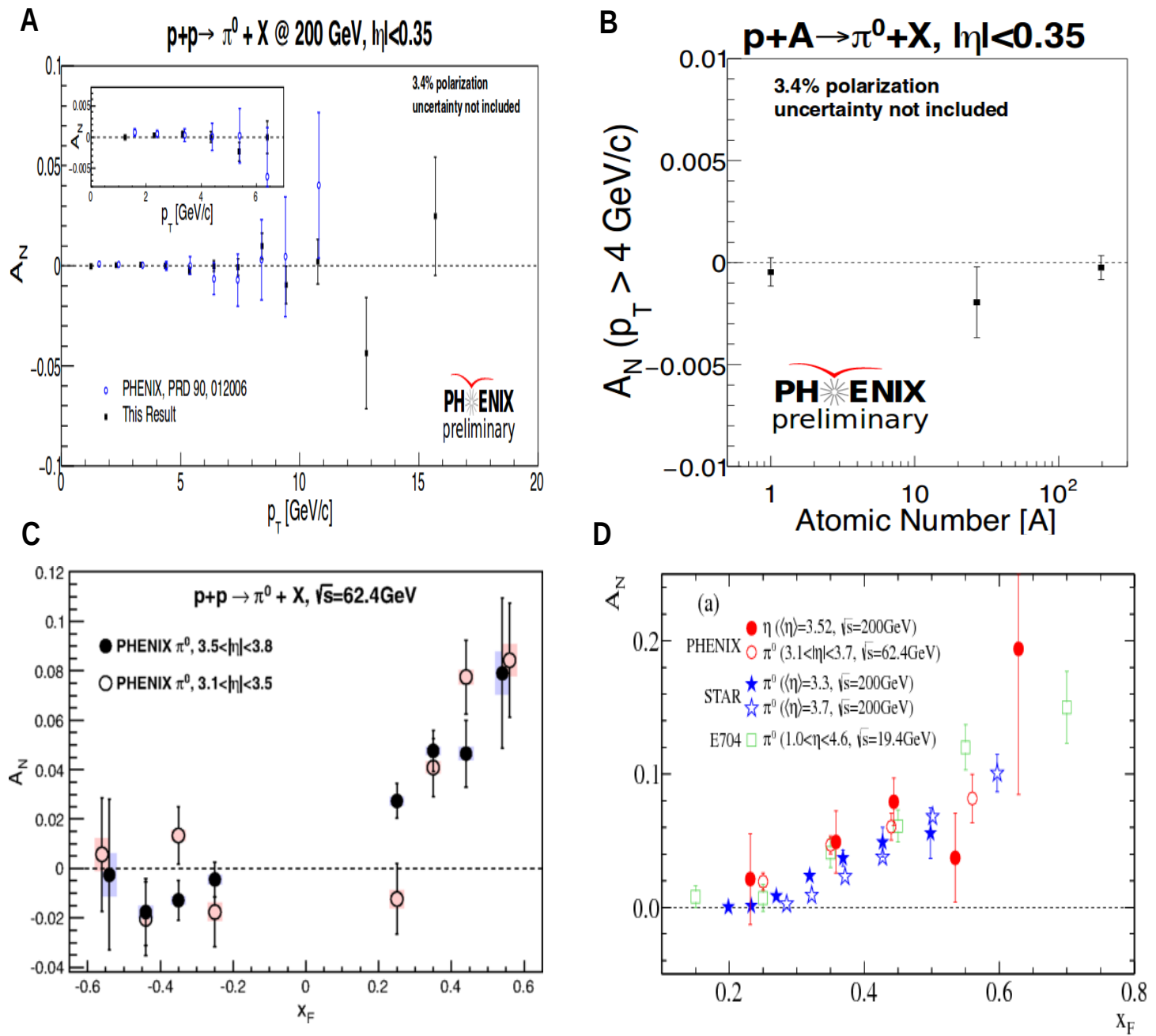

Figure 1: Top left panel (A, from [7]): $\mathrm{A}_{N}$ measured at midrapidity $(|\eta|<0.35)$, as function of $\mathrm{p}_{T}$ for $\pi^{0}$. Latest PHENIX results from 2015 data (black squares) are compared to [8] (blue open dots). Top right panel (B, from [7]): nuclear dependence of the integrated $\mathrm{A}_{N}$ for $\mathrm{p}_{T}>4 \mathrm{GeV} / \mathrm{c}$. Bottom left panel (C from [8]): Neutral pion $\mathrm{A}_{N}$ at $\sqrt{s}=62.4 \mathrm{GeV}$ as function of $\mathrm{x}_{F}$ in two different pseudorapidity ranges $(3.1<|\eta|<3.5$ and $3.5<|\eta|<3.8)$ with statistical and systematic uncertainties. Bottom right panel (D from [9]): Comparison between the $\eta$ meson $\mathrm{A}_{N}$ and $\pi^{0}$ meson $\mathrm{A}_{N}$ results from PHENIX [8], STAR [10], and E704 [11] in red circle, blue star, and green square symbols, respectively. For all figures, an additional uncertainty from the beam polarization is not included.

Asymmetries in hadron production at central rapidity are expected to be related to the moments of the gluon Sivers function [1]. Fig. 1 (top left panel) displays the latest measurement of the $\pi^{0} A_{N}$ at mid-rapidity [7, 8]. The results were found to be consistent with zero for all bins over an extended $\mathrm{p}_{T}$ range. Recently, the $\pi^{0} A_{N}$ was also measured in $\mathrm{p}+\mathrm{A}$ collisions and no quantifiable 
nuclear dependence was found within uncertainties (Fig. 1, top right panel) [7].

The same measurement was also performed at forward and backward rapidities $(3.1<|\eta|<$ 3.8 ) at an energy of $62.4 \mathrm{GeV}$. Fig. 1 (bottom left panel) displays the measured asymmetry as a function of $x_{F}$. Whereas the backward asymmetry is consistent with zero, we found large asymmetries in the forward direction. The $A_{N}$ has a clear dependence and is increasing as a function of $x_{F}$ and $p_{T}$. In order to check for energy dependence, a comparison has been made with other experiments which did a similar measurement. The asymmetries are found to be nearly independent of the collision energy (Fig. 1, bottom right panel).

A similar measurement has been done for the $\eta$ meson [9], and similar results were found. Indeed, the large asymmetries found in the forward region are at the same level as those for $\pi^{0}$ production (Fig. 1, bottom right panel). Similarly, the $A_{N}$ was found to be consistent with zero in the backward region and was found to be increasing as a function of $p_{T}$ in the forward direction. Observations lead to the conclusion of a weak energy dependence for both $A_{N}$ in $\pi^{0}$ and $\eta$ production, with no quantifiable difference for both measurements. Twist- 3 calculations using quark-gluon correlation functions manage to reproduce the experimental result [12]. However, the interpretation of these results in term of nucleon transverse structure is still subject of discussions.

\section{Transverse spin asymmetries for muons from heavy flavor decays}

The measurement of this asymmetry would allow for discriminating between various models. The production mechanism of heavy flavored mesons is largely dominated by gluon-gluon interactions. Non-zero transverse spin asymmetries in the production of heavy meson would arise from initial state effects, and are expected to be large for the case of a large gluon correlation function, associated to the gluon Sivers function [13]. Recently, $A_{N}$ for open charm production, reconstructed as single muon decays, and dominated by D meson production were published [14]. The measurement was performed in the forward and backward rapidity regions $(1.2<|\eta|<2.2)$ at $\sqrt{s}=200 \mathrm{GeV}$. Whereas the measured asymmetries are found to be consistent with zero (Fig. 2 ), a comparison between $\mu^{+}$and $\mu^{-}$production was performed as well as kinematic dependence. We found a tendency for a small $x_{F}$ dependence for decays into $\mu^{+}$(dominated by $\mathrm{D}^{+}$meson production). Model calculations including twist-3 effect, within a collinear factorization framework are reproducing this tendency $[15,16]$.

\section{Transverse spin asymmetries of $\mathrm{J} / \Psi$ mesons}

According to [17], asymmetries in transversely polarized $\mathrm{J} / \Psi$ production are sensitive to the $\mathrm{J} / \Psi$ production mechanism and allow us to discriminate between different models. Larger asymmetries are expected for a dominant color singlet model, coming from initial state interactions, while smaller asymmetries would be expected for a dominant color octet model, as both initial and final state effects would occur that cancel asymmetry effects. Four data sets were analyzed for this measurement, with a huge increase $(\times 5)$ of available statistics for the most recent run. Fig. 3 

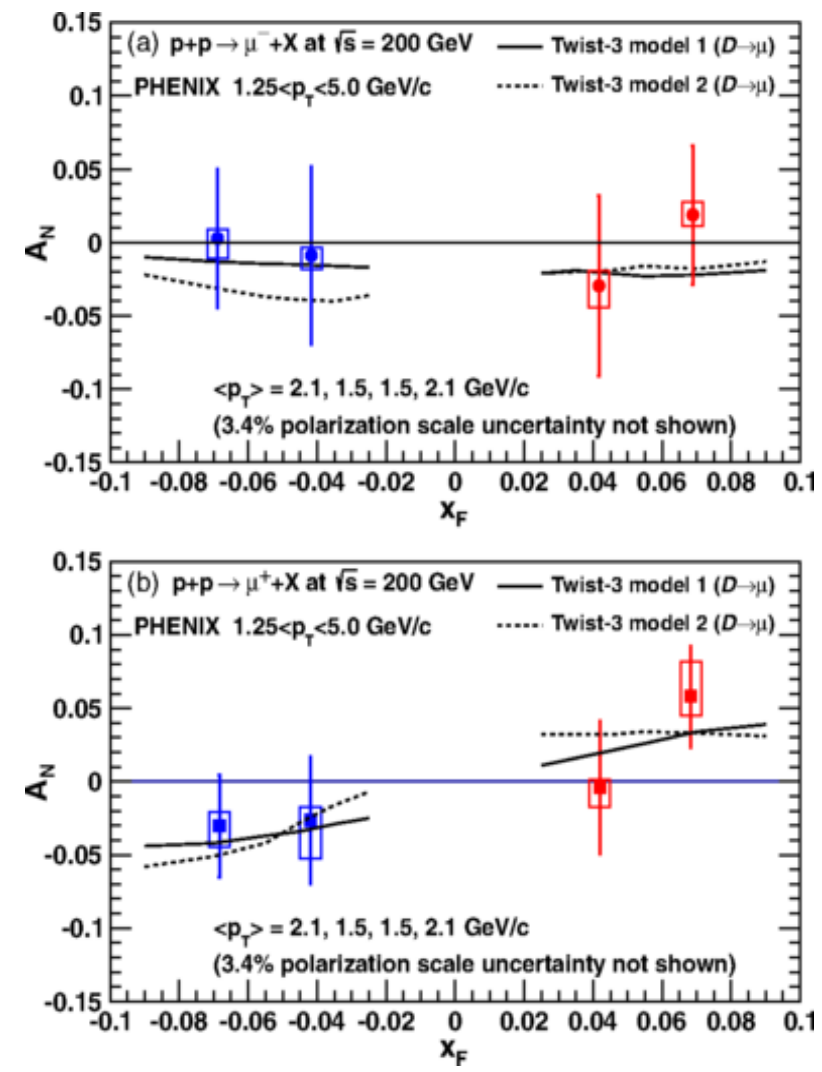

Figure 2: $A_{N}$ of (top panel) negatively charged and (bottom panel) positively charged muons from open heavy-flavor decays as a function of $\mathrm{x}_{F}$, where $\mathrm{x}_{F}>0$ is along the direction of the polarized proton. Vertical bars (boxes) represent statistical (systematic) uncertainties. Solid and dashed lines represent twist-3 model calculations [16].
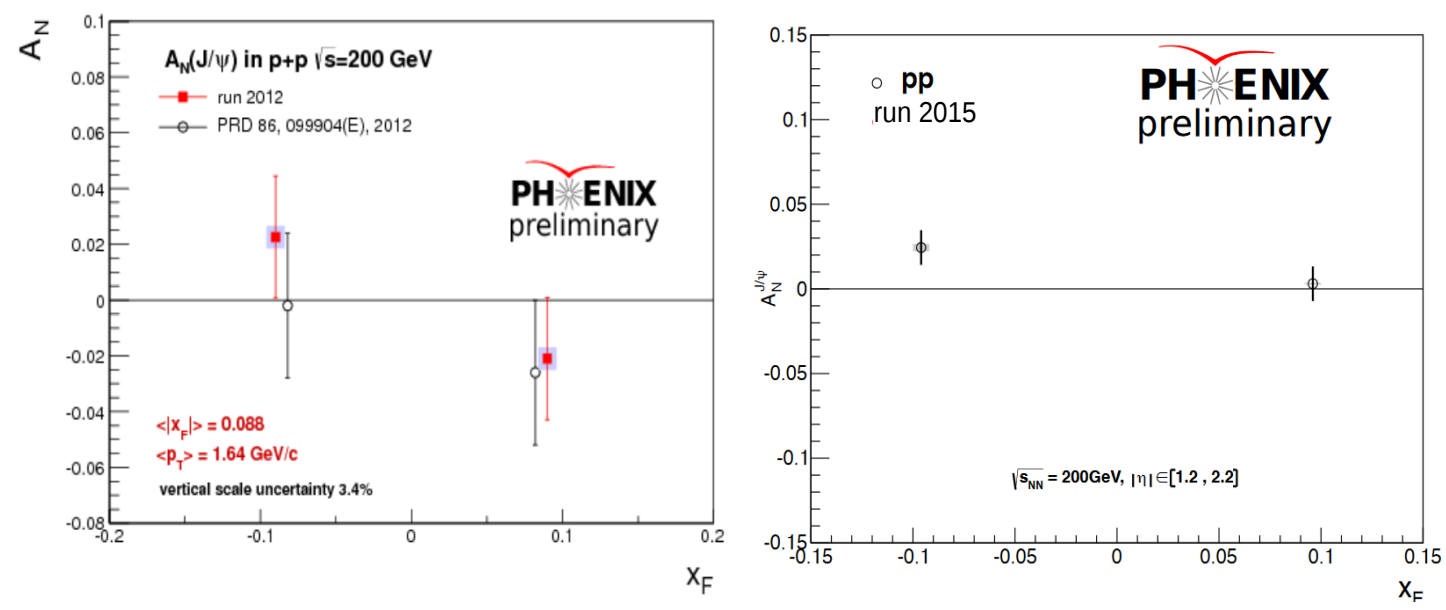

Figure 3: Transverse spin asymmetry in $J / \Psi$ production in $\mathrm{p}+\mathrm{p}$ collisions at $\sqrt{s}=200 \mathrm{GeV}$ as a function of $x_{F}$. Left panel: analysis results from combined 2006+2008 data sets compared to the 2012 data set $[18,19]$. Right panel: results from the 2015 data set [20]. 
presents comparisions of these various measurements and dependencies as a function of $x_{F}$ (backward and forward). With improved statistics, the measured asymmetry is still consistent with zero, within uncertainties $[18,19,20]$.

\section{Summary}

We presented a summary of recent measurements of transverse spin asymmetries $A_{N}$ in the PHENIX experiment. Several years of data allow for a broad range of measurement of such asymmetries, enabling a better understanding of the nucleon's transverse structure. New exciting results are upcoming with further analysis. In particular, the first proton-ion data have recently been taken which allow for the first time to study nuclear effects in $A_{N}$ measurements.

\section{References}

[1] E. C. Aschenauer et al., arXiv:1501.01220 [nucl-ex].

[2] D. W. Sivers, Phys. Rev. D 41, 83 (1990).

[3] J. C. Collins, Nucl. Phys. B 396 (1993) 161

[4] D. Boer and P. J. Mulders, Phys. Rev. D57, 5780 (1998).

[5] Run overview of the Relativistic Heavy Ion Collider http://www.agsrhichome.bnl.gov/RHIC/Runs/

[6] K. Adcox et al. [PHENIX Collaboration], Nucl. Instrum. Meth. A 499 (2003) 469.

[7] N. Novitzky, presentation, 22nd International Spin Symposium (2016).

[8] A. Adare et al. [PHENIX Collaboration], Phys. Rev. D 90 (2014) no.1, 012006

[9] A. Adare et al. [PHENIX Collaboration], Phys. Rev. D 90 (2014) no.7, 072008

[10] B. I. Abelev et al. [STAR Collaboration], Phys. Rev. Lett. 101 (2008) 222001

[11] D. L. Adams et al. [E581 and E704 Collaborations], Phys. Lett. B 261 (1991) 201.

[12] K. Kanazawa and Y. Koike (2011), private communication to PHENIX.

[13] Z. B. Kang, J. W. Qiu, W. Vogelsang and F. Yuan, Phys. Rev. D 83 (2011) 094001

[14] C. Aidala et al., Phys. Rev. D 95 (2017) no.11, 112001

[15] Y. Koike and S. Yoshida, Phys. Rev. D 84 (2011) 014026

[16] S. Yoshida, private communication.

[17] F. Yuan, C. F. Qiao and K. T. Chao, Phys. Rev. D 56 (1997) 321

[18] A. Adare et al. [PHENIX Collaboration], Phys. Rev. D 82 (2010) 112008 Erratum: [Phys. Rev. D 86 (2012) 099904]

[19] O.K. Eyser, PoS DIS2013 (2013) 241.

[20] C. Xu, presentation, 22d International Spin Symposium (2016). 